\title{
Sprache als Werkzeug des Lernens: Ein Überblick zu den kommunikativen und kognitiven Funktionen der Sprache und deren Bedeutung für den fachlichen Wissenserwerb
}

\author{
Sebastian Kempert ${ }^{1}$, Lennart Schalk², Henrik Saalbach ${ }^{3}$ \\ Universität Potsdam \\ ETH Zürich \& PH Schwyz \\ Universität Leipzig
}

\begin{abstract}
Zusammenfassung: Der enge Zusammenhang von sprachlichen Kompetenzen und fachlichem Lernen ist empirisch gut belegt. Ebenfalls umfassend untersucht und beschrieben sind die vielfältigen Wechselwirkungen zwischen sprachlichen und kognitiven Prozessen, dies gilt insbesondere für das Kindesalter. Bisher liegt jedoch keine integrierende Übersicht über die Zusammenhänge von sprachlichen und kognitiven Funktionen sowie fachlichem Lernen im Schulkontext vor. Dieser Beitrag bietet einen Überblick, wie sich Sprache und kognitive Prozesse in ihrer Entwicklung gegenseitig beeinflussen und welche Konsequenzen sich daraus für das fachliche Lernen in der Schule ableiten lassen. Auf der Basis der dargestellten Befunde wird dann skizziert, welche Prinzipien im fachlichen Unterricht berücksichtigt werden sollten, um sprachliche und fachliche Lerngelegenheiten zu verbinden. Sprache kann sich so zu einem immer besseren Werkzeug des Lernens entwickeln. Sprachliche Kompetenzen in ihrer kommunikativen und kognitiven Funktion sind in diesem Sinne also nicht nur Voraussetzung, sondern auch Ziel fachlichen Lernens.
\end{abstract}

Schlüsselbegriffe: Sprache, fachliches Lernen, Kognition

\section{Language as Tool for Learning: A Review of the Communicative and Cognitive Functions of Language and its Relevance for Academic Learning}

Summary: There is considerably evidence for the close relationship between language proficiency and academic (content specific) learning. Moreover, research has shown manifold interactions between language and cognitive processes especially during early childhood. But, an integrative overview of how language, cognitive processes, and academic learning interact is lacking. In the present article, we review how language and cognition mutually affect each other in development and how this interplay relates to content knowledge acquisition in school. Based on the reviewed empirical studies, we outline which principles have to be implemented in instruction in order to address language development and content learning simultaneously. Language thus becomes a continuously improving tool for learning. Consequently, the communicative and the cognitive functions of language cannot only be considered a prerequisite but also an objective in content knowledge acquisition.

Keywords: Language, academic learning, cognition

Lehr- und Lernprozesse sind ohne Sprache nicht vorstellbar. Sprache spielt eine zentrale Rolle nicht nur bei der Wissensvermittlung und der individuellen Wissenskonstruktion, sondern auch beim Abruf von
Gelerntem und dem Verständnis von Testaufgaben. Sprache wird folglich als notwendige Ressource für die schulische und akademische Entwicklung aufgeführt (z. B. Kempert et al., 2016). 
Die Bedeutung sprachlicher Kompetenzen für den Erwerb bereichsspezifischen Wissens und fachlicher Kompetenzen (nachfolgend nutzen wir hierfür die Bezeichnung fachliches Lernen) wird vor allem dann hervorgehoben, wenn Muttersprache und Instruktionssprache nicht identisch sind. Das ist beispielsweise dann der Fall, wenn Deutsch die Instruktionssprache ist, die Schülerin oder der Schüler mit Zuwanderungshintergrund jedoch Deutsch als Zweitsprache spricht (z. B. Kempert, Saalbach \& Hardy, 2011; Saalbach, Gunzenhauser, Kempert \& Karbach, 2016) oder wenn im Rahmen von Immersionsunterricht auf Englisch unterrichtet wird, Deutsch aber die Muttersprache der meisten Schülerinnen und Schüler ist (z. B. Grabner, Saalbach \& Eckstein, 2012; Möller, Hohenstein, Fleckenstein \& Baumert, 2017; Saalbach, Eckstein, Andri, Hobi \& Grabner, 2013). Im vorliegenden Beitrag wird dafür argumentiert, dass es auch über diese spezifischen Kontexte hinaus essenziell ist, die Beziehung zwischen Sprache und fachlichem Lernen in der Beschreibung und Untersuchung von Lehrund Lernprozessen zu berücksichtigen. Hierfür gibt es zwei Gründe. Erstens ist die sprachliche Entwicklung der Kinder bei Eintritt in die Schule nicht abgeschlossen (vgl. Ehlich, Bredel $\&$ Reich, 2008). Zweitens interagiert Sprache mit basalen kognitiven Prozessen bei der Wissensaneignung. Letzteres trifft überdies nicht nur auf Kinder, sondern auch auf Erwachsene zu (vgl. Gentner, 2016).

Im Folgenden zeichnen wir zunächst den engen Zusammenhang von sprachlicher und kognitiver Entwicklung im Kindesalter nach. Zudem stellen wir Studien dar, die belegen, dass kognitive Prozesse der Wissensaneignung - wie beispielsweise das schlussfolgernde Denken sensitiv für sprachliche Variationen sind. Darauf aufbauend erläutern wir, welche Bedeutung diese Wechselwirkungen für die Wissensvermittlung und das fachliche Lernen haben (z. B. im naturwissenschaftlichen und mathematischen Unterricht). In diesem Zusammenhang gehen wir außerdem mittels eines Exkurses auf Befunde zum bilingualen Lernen bzw. fremd- sprachlichen Fachunterricht ein, da diese Unterrichtsform die gleichzeitige Berücksichtigung sprachlicher und fachlicher Kompetenzen explizit erfordert. Diese integrierende Betrachtung erlaubt es abschließend, allgemeine Prinzipien abzuleiten, die bei der Planung und Durchführung von Unterricht berücksichtigt werden sollten, um sowohl die sprachliche Entwicklung als auch das fachliche Lernen zu unterstützen.

\section{Der Zusammenhang von sprachlicher und kognitiver Entwicklung im Kindesalter und dessen Auswirkungen auf den Wissenserwerb}

Trotz ihrer konzeptuellen Trennbarkeit weisen die kognitive und die sprachliche Entwicklung zahlreiche Abhängigkeiten auf. Je nach Alter und Entwicklungsstand nehmen diese Abhängigkeiten unterschiedliche Wirkrichtungen an (Weinert, 2016). Einerseits bilden kognitive Prozesse die Voraussetzungen für den Spracherwerb und die Sprachnutzung. Andererseits beeinflusst die Art der sprachlichen Formulierung (z. B. in Texten, in Aufgabenstellungen und in Instruktionen durch die Lehrperson) die kognitive Verarbeitung und Nutzung der dargebotenen Information.

\section{Spracherwerb und kognitive Entwicklung}

Zu Beginn des Spracherwerbs muss das sprachliche Angebot der Umwelt wahrgenommen, analysiert, kategorisiert und gespeichert werden. Kindern gelingt diese anspruchsvolle Entwicklungsaufgabe, indem sie Hinweisreize auf unterschiedlichen Ebenen nutzen (z. B. HirshPasek \& Golinkoff, 1999). Der Stellenwert dieser Hinweisreize variiert im Verlauf von der kindlichen Entwicklung. Zu Beginn des Spracherwerbs spielen zunächst prosodische Aspekte eine zentrale Rolle, darauf folgen semantische Aspekte. Mit fortschreitender kognitiver Entwicklung können Kinder dann syntaktische 
sowie soziale Hinweisreize nutzen, um sprachliche Äußerungen zu verstehen. Die zunehmend integrierte Nutzung verschiedener Hinweisreize ermöglicht es Kindern schließlich, generelle Regelmäßigkeiten der Sprache zu erkennen, diese nachzuvollziehen und Sprache selbstständig zu (re)produzieren sowie generativ - also flexibel und kreativ - anzuwenden (Weinert, 2010, 2016).

Insbesondere zu Beginn des Spracherwerbs, wenn es um das Erlernen von Lauten, Wörtern und einfachen Sprachstrukturen geht, gilt die phonologische Schleife des Arbeitsgedächtnisses als zentrale Ressource (z. B. Gathercole \& Baddeley, 1993). Die phonologische Schleife ermöglicht das kurzzeitige Halten und Wiederholen einer begrenzten Menge von sprachlichen Informationen. Gerade wenn noch kein Netzwerk phonologisch-lexikalisch ähnlicher Wörter aufgebaut ist, muss jedes Wort ressourcenintensiv im Arbeitsgedächtnis repräsentiert und analysiert werden, um es letztlich in das Langzeitgedächtnis zu integrieren. Mit anderen Worten: Weist ein neuer Laut oder ein neues Wort keine klangliche oder bedeutungsbezogene Ähnlichkeit mit schon bekannten Lauten oder Wörtern auf, ist die Arbeitsgedächtnisbelastung höher als bei entsprechender Ähnlichkeit. Somit ist zu Beginn des Spracherwerbs das Arbeitsgedächtnis der kognitive Flaschenhals, der durch seine Begrenztheit bestimmt, wie viel Information aus der Umwelt aufgenommen werden kann.

Mit zunehmender Enkodierung und Repräsentation von phonologisch-lexikalischem Wissen ändert sich jedoch die Wirkrichtung: Das im Langzeitgedächtnis repräsentierte sprachliche Wissen beeinflusst nun die phonologische Informationsverarbeitungskapazität des Arbeitsgedächtnisses (Gathercole, Willis, Emslie \& Baddeley, 1992). Dieses Zusammenspiel zwischen Arbeits- und Langzeitgedächtnis lässt sich tatsächlich nicht nur zu Beginn des Erstspracherwerbs identifizieren. Auch wenn ältere Kinder oder Erwachsene weitere Sprachen erlernen, spielt die phonologische Schleife zunächst eine sehr wichtige Rolle. Dies ist vor allem dann der Fall, wenn sich Erst- und Zweitsprache phonotaktisch, d. h. in der Art typischer Lautverbindungen einer Sprache, bedeutsam unterscheiden (z. B. Service \& Kohonen, 1995). Erst in späteren Phasen des Spracherwerbs bzw. des sprachlichen Handelns wird dann das bereichsunspezifische Arbeitsgedächtnis (zentrale Exekutive) immer wichtiger, wenn es beispielsweise um die Bedeutungserschließung unbekannter Wörter aus dem linguistischen und sozio-pragmatischen Kontext geht (Daneman \& Green, 1986).

Die enge Wechselwirkung zwischen sprachlichen Kompetenzen und dem Arbeitsgedächtnis als grundlegender kognitiver Prozess ist auch durch Befunde aus der Gedächtnispsychologie belegt. So werden Stimuli besser erinnert, wenn sie zusätzlich zur nicht-sprachlichen Ausgangsmodalität (z. B. Präsentation eines Bildes) sprachlich benannt werden (für einen Überblick siehe Weinert, 2010). Sprache wird zu einer noch stärkeren Hilfe für das Lernen, wenn neben dem einfachen Benennen auch Strategien der sprachlichen Elaboration oder Strukturierung angewendet werden, die beispielsweise dazu anregen, einen neuen Sachverhalt in eigene Worte zu fassen (Renkl, 2009). Aufgrund dieser Befunde kann angenommen werden, dass für fachliche Konzepte, für die noch kein geeigneter Wortschatz aufgebaut wurde, das Lernen erschwert ist.

\section{DerEinfluss spezifischer Sprachstrukturen auf den Wissenserwerb und das schlussfolgernde Denken}

Nicht nur grundlegende kognitive Prozesse stehen in einer wechselseitigen Beziehung zu Sprachkompetenzen, sondern auch höhere kognitive Prozesse. Vygotsky (2012) zufolge hat Sprache einen transformativen Einfluss auf die kognitiven Fähigkeiten des Kindes (vgl. auch Tomasello, 1999). Das Lernen einer Sprache führt demnach zu einer dramatischen Entwicklung der vorsprachlichen kognitiven Fähigkeiten des Kindes hin zu neuen Kompetenzen, wie 
etwa fokussierter Aufmerksamkeit, erleichterter Enkodierung und effizienterem Abruf von Gedächtnisinhalten. Diese Kompetenzen versetzen das Kind zudem in die Lage, die eigenen mentalen Prozesse und Ressourcen besser zu kontrollieren.

So führt beispielsweise das Erlernen der Zahlwörter in der frühen Kindheit zur Integration angeborener numerischer Fähigkeiten (der sog. number senses: die exakte Repräsentation kleiner Mengen und die ungefähre Repräsentation großer Mengen; z. B. Dehaene, 1997; Dehaene, Spelke, Pinel, Stranescu \& Tsivkin, 1999). Die Integration von angeborenen Fähigkeiten und erlernten fachlichen Fertigkeiten bildet dann die Grundlage für die Entwicklung höherer mathematischer Kompetenzen (Gordon, 2004; Pica, Lemer, Izard \& Dehaene, 2004). Der Einfluss der Sprache auf die Entwicklung mathematischer Kompetenzen wird zudem durch Studienergebnisse unterstrichen, die strukturelle Unterschiede zwischen Zahlensystemen verschiedener Sprachen mit bestimmten Entwicklungsverläufen im Hinblick auf grundlegende Zähl- und Rechenfähigkeit in Zusammenhang bringen (Miller, 1996; Miller, Smith, Zhu \& Zhang, 1995). Ist ein Zahlensystem einfach und kohärent aufgebaut (wie z. B. im Chinesischen), lernen Kinder schneller zählen, als wenn ein Zahlensystem verschiedene unregelmäßige Charakteristika aufweist (wie z. B. im Englischen oder Französischen).

Ein weiteres Beispiel für die Wechselwirkung von Sprache und höheren kognitiven Prozessen betrifft das Erlernen bzw. die Bildung von taxonomischen Kategorien: Das explizite sprachliche Benennen von Objekten durch Erwachsene hilft Kindern am Anfang des Spracherwerbs (im Alter von ca. 12 bis 13 Monaten), übergeordnete Kategorien zu bilden (z. B. Waxman \& Markow, 1995). Wenn die Kinder in entsprechenden Experimenten verschiedene Mitglieder einer Kategorie (z. B. Tiere) sahen und währenddessen der entsprechende Kategorienamen genannt wurde (z. B. „Schau mal dieses Tier an!"), hatten sie einen deutlichen Vorteil bei der induktiven Bildung von Kategorien im Vergleich zu Kindern, denen nur das Mitglied der Kategorie ohne Nennung des Kategorienamens gezeigt wurde. Eine Kategorienbezeichnung hilft Kindern zudem, Wissen von einem bekannten Exemplar der Kategorie deduktiv auf ein neues, unbekanntes Exemplar zu übertragen, das laut der sprachlichen Bezeichnung derselben Kategorie angehört. Kinder im Vorschulalter nehmen also offensichtlich an, dass Objekte mit demselben Kategorienamen wichtige Eigenschaften teilen (Gelman \& Markman, 1986; Imai \& Saalbach, 2007; Imai, Saalbach \& Stern, 2010). Gleichzeitig können aber spezifische Charakteristika einer Sprache zu Verzerrungen führen. Auf den ersten Blick recht unbedeutende linguistische Marker wie das grammatikalische Geschlecht können Kinder leiten, wenn sie Eigenschaften über Exemplare verallgemeinern (z. B. Saalbach, Imai \& Schalk, 2012) - auch bei Erwachsenen sind diese Effekte noch zu beobachten (Imai, Schalk, Saalbach \& Okada, 2014). Wird beispielsweise weiblichen Tieren eine Eigenschaft zugeschrieben, neigen Kinder und Erwachsene dazu, diese Eigenschaft fälschlicherweise eher auf die (grammatikalisch feminine) Giraffe als auf den (grammatikalisch maskulinen) Elefanten zu verallgemeinern. Durch diese Effekte wird deutlich, wie eng das Lernen und die Nutzung von Kategorien mit der Sprache gekoppelt sind.

Auch die Forschung zum analogen SchlieBen unterstreicht die Bedeutung von (relationaler) Sprache für das Lernen. Nach Gentner (2003, 2010; siehe auch Gentner \& Hoyos, 2017) ist das analoge Schließen eine der mächtigsten Triebfedern für intellektuelle Leistungen. Analoges Schließen bezeichnet die Fähigkeit, durch Vergleiche zwischen zwei oder mehr Repräsentationen grundlegende Gemeinsamkeiten und Unterschiede zu erkennen. Bei hinreichender struktureller oder konzeptueller Ähnlichkeit zwischen den Repräsentationen (z. B. Hund und Delfin als Mitglieder der taxonomischen Kategorie Säugetiere) können Informationen von der einen auf die andere übertragen werden. Solche Vergleichsprozesse ermöglichen es nicht nur, die Eigenschaften einer zuvor unbekannten Reprä- 
sentation zu erschließen, sondern auch ein allgemeines, abstraktes Prinzip (z. B. „Säugetiere säugen ihren Nachwuchs") abzuleiten, das die zu vergleichenden Repräsentationen verbindet. Die Ableitung eines Prinzips bildet damit die Grundlage für den Transfer des Gelernten (Schalk, Saalbach \& Stern, 2016). Erfährt ein Kind bei einem Zoobesuch, dass ein ihm bisher unbekanntes Tier ein Säugetier ist, kann das Kind daraus schließen, dass dieses Tier wohl den Nachwuchs säugen und weitere typische Merkmale von Säugetieren aufweisen wird.

Die Art, wie verschiedene Repräsentationen oder Situationen sprachlich eingebettet werden, beeinflusst direkt die Verständnis- und Transferleistung. Studien zum analogen Schlussfolgern von jungen Kindern zeigen eindrucksvoll, wie kleine sprachliche Hinweise die Leistungen von Vorschulkindern verändern. Werden Kinder beispielsweise bei räumlichen Zuordnungsaufgaben instruiert, durch einen Vergleich zweier vorgegebener Versuchsanordnungen mit jeweils drei Objekten aufsteigender Größe das analoge Zielobjekt zu identifizieren, ist der Erfolg stark abhängig von der sprachlichen Einbettung der Aufgabe (Loewenstein \& Gentner, 1998; ebenso Gentner, 2010). Die korrekte Lösung des Problems wird durch das Erkennen der relationalen Ähnlichkeit (hier die aufsteigende Größe) der Objekte ermöglicht. Werden die Objekte mit relationalen sprachlichen Etiketten versehen (z. B. winzig, klein, groß), erhöhen sich die Leistungen (korrekte Identifikation der analogen Versuchsanordnung) der Kinder deutlich im Vergleich zu einer Bedingung, in der die Relationen nicht sprachlich benannt werden. Die Wirkung relationaler Sprache hat sich als zeitlich stabil erwiesen und tritt selbst dann noch auf, wenn in entsprechenden Posttests die relationalen sprachlichen Bezeichnungen nicht erneut verwendet werden. Die Nutzung relationaler Sprache wirkt also nicht nur in der konkreten Situation unterstützend, sondern ermöglicht zudem - wie oben bereits erwähnt - die Ableitung eines allgemeinen Prinzips, das dann bei ähnlichen Aufgabenstellungen flexibel und spontan verwendet werden kann.
Zusätzlich zur Benennung von Kategorien und der Verwendung relationaler Sprache wirkt sich auch der Gebrauch generischer Sprachkonstruktionen deutlich auf das schlussfolgernde Denken junger Kinder aus (Cimpian \& Markman, 2008). So macht es einen Unterschied, ob ein Kind anhand einer generischen Sprachkonstruktion etwas über eine Entität im Allgemeinen erfährt („Katzen jagen Mäuse“) oder die Sprachkonstruktion lediglich etwas über ein Individuum ausdrückt („Nachbars Katze jagt Mäuse“). Generische Sprachkonstruktionen aktivieren einerseits das mit der jeweiligen Kategorie verbundene, zentrale konzeptuelle Wissen, andererseits vermitteln sie auch eben dieses für eine Kategorie zentrale Wissen. Mit einer Kategorie (z. B. Katzen) sind bestimmte Kerneigenschaften oder Ideale assoziiert, während individuelle Instanzen oder Exemplare einer Kategorie (die Katze des Nachbarn) darüber hinaus immer auch mit idiosynkratrischen Eigenschaften verbunden sind (z. B. ihr Fell ist gestreift und sie miaut nachts laut). Individuelle Exemplare weisen somit eine höhere Variabilität ihrer Merkmale auf; gleichzeitig sind nicht alle ihre Merkmale charakteristisch für eine Kategorie. Generische und spezifische sprachliche Formulierungen könnten demnach im Lehr-Lern-Prozess je nach aktuellem Fokus gezielt eingesetzt werden. Sollen generelle charakteristische Eigenschaften einer Kategorie gelernt werden, sollte die Information anders formuliert werden, als wenn es um die Beschreibung eines ganz bestimmten Elements einer Kategorie mit ggf. abweichenden Merkmalen geht (Gelman, Ware, Manczak \& Graham, 2013).

Neben generischen Konstruktionen (z. B. die Katzen) wirkt sich auch die sprachliche Verwendung von Quantoren (alle Katzen, einige Katzen) auf die Variabilität in den zugewiesenen Eigenschaften der jeweiligen Art aus. Quantoren drücken aus, wie weitreichend eine Aussage ist, und tatsächlich beeinflussen diese Angaben auch die Reichweite der gezogenen Schlussfolgerungen (z. B. Gelman, Star \& Flukes, 2002). 
Eng mit den gerade geschilderten Befunden verknüpft ist ein weiterer Aspekt, der durch Sprache beeinflusst wird - die Tiefe der Verarbeitung bei Vergleichsprozessen. Die Art der sprachlichen Einbettung von Aufgaben, in denen verschiedene Beispiele oder Exemplare verglichen werden, lenkt den Fokus der Aufmerksamkeit. Diese sprachliche Aufmerksamkeitslenkung bestimmt, welche Eigenschaften vornehmlich wahrgenommen werden. Damit hat sie einen direkten Einfluss auf die Kategorienbildung und davon beeinflusste Schlussprozesse (Gelman, Raman \& Gentner, 2009). Variiert man z. B. bei Vergleichen die Ähnlichkeit der Objekte (Hunde und Katzen versus Hunde und Giraffen) und die sprachliche Einbettung (generisch versus spezifisch), zeigt sich, dass die Kombination aus hoher Ähnlichkeit und generischer Formulierung zu konzeptuell tieferen Vergleichen führt. Das heißt, dass Kinder, aber auch Erwachsene mehr Aussagen über Eigenschaften produzieren, die nicht direkt beobachtbar sind (z. B. „Beide sind Säugetiere!“), als Aussagen, die sich auf unmittelbare Oberflächenmerkmale beziehen (z. B. „Beide haben ein Fell!"). Zusammenfassend lässt sich vor dem Hintergrund der in diesem Abschnitt dargelegten Befunde feststellen, dass die Sprachverwendung höhere kognitive Prozesse wie das induktive, deduktive und analoge Schließen und das Kategorienlernen deutlich beeinflussen kann.

\section{Selbstinstruktion als kognitives Werkzeug}

In den im vorherigen Abschnitt erwähnten Studien wurde Sprache als Medium der Wissensvermittlung in Situationen eingesetzt, in denen zwei oder mehr Personen miteinander interagieren und Sprache demzufolge von außen an die Rezipienten herangetragen wird. Sprache spielt aber auch bei der Modulation und Regulation kognitiver Prozesse eine wichtige Rolle, wenn sie sich an einen selbst richtet
(Vygotsky, 1987). Kinder sprechen häufig mit sich selbst, wenn sie sich intensiv mit einer Aufgabe oder einem Spiel beschäftigen. Diese Selbstgespräche treten im Alter von fünf Jahren am häufigsten auf. Danach werden sie allmählich in eine Form des inneren Sprechens überführt, welche nur noch durch Flüstern, Murmeln oder Lippenbewegungen erkennbar ist, bis es schließlich im Grundschulalter kaum mehr äußere Anzeichen des inneren Sprechens gibt (Winsler, 2009; Winsler \& Naglieri, 2003).

Kinder lernen diese regulative Funktion von Sprache durch Eltern sowie Erzieherinnen und Erzieher kennen, wenn diese verbale Strategien einsetzen, um die soziale Interaktion und das Verhalten der Kinder zu kommentieren und dadurch zu modulieren. In einem Prozess der zunehmenden Internalisierung verwenden Kinder diese Strategien mehr und mehr für sich selbst, indem sie zunächst zu sich selbst wie zu einer anderen Person sprechen und schließlich zum inneren Sprechen übergehen. Neuere Studien zur Entwicklung von Selbstgesprächen deuten darauf hin, dass Kinder mit besseren verbalen Fähigkeiten auch mehr an sich selbst gerichtete Sprache einsetzen, um Probleme und Aufgaben zu lösen (Fernyhough \& Fradley, 2005; Vallotton \& Ayoub, 2011). Studien auf Basis von Selbstberichten oder Interferenzparadigmen, bei denen zwei Aufgaben mit ähnlichen Anforderungen gleichzeitig bearbeitet werden müssen, weisen darauf hin, dass verbale Strategien während der Bearbeitung anspruchsvoller Aufgaben auch bei älteren Kindern und Erwachsenen noch zum Einsatz kommen (Lidstone, Meins \& Fernyhough, 2010; Vygotsky, 1987; Winsler \& Naglieri, 2003). Vor dem Hintergrund der angenommenen Wichtigkeit verbaler Strategien bei verschiedenen kognitiven Anforderungen wie etwa Planungsaufgaben (z. B. Alderson-Day \& Fernyhough, 2015; Perrone-Bertolottia, Rapin, Lauchaux, Baciu \& Lovenbruck, 2014; Vygotsky, 1987) stellt sich die Frage, welche Konsequenzen es hat, wenn beispielsweise auf- 
grund von Sprachdefiziten weniger Selbstgespräche bzw. Selbstinstruktionen eingesetzt werden können (z. B. Gunzenhauser, Hauch, Stephan \& Saalbach, in Druck). Hierzu sind bislang jedoch kaum Forschungsarbeiten veröffentlicht und es lassen sich noch keine belastbaren Aussagen treffen. Vermutlich wirken sich jedoch Einschränkungen in der Verwendung von an sich selbst gerichteter Sprache auch negativ auf die Nutzung von lernwirksamen Strategien der sprachlichen Elaboration (z. B. das Umformulieren eines Sachverhalts in eigene Worte) aus.

\section{Die Bedeutung von Sprache für das fachliche Lernen}

Die eben beschriebenen Interaktionen von Sprache und grundlegenden sowie höheren kognitiven Prozessen stammen größtenteils aus experimentellen Laboruntersuchungen, die gezielt auf die genaue Betrachtung einzelner Prozesse und isolierter Zusammenhänge ausgelegt wurden. Aufgrund dieser Befunde scheint es aber naheliegend, dass sich auch bei komplexeren Lernprozessen - wie dem fachlichen Lernen, bei dem mehrere grundlegende und höhere kognitive Prozesse gleichzeitig genutzt oder koordiniert werden müssen - Wechselwirkungen mit Sprache zeigen sollten. Neben der Prüfung des generellen Zusammenhangs von sprachlichen und fachlichen Kompetenzen ist die Identifizierung möglicher Wirkzusammenhänge zentral. In diesen Zusammenhang ist auch der Blick auf bilinguale Lernkontexte aufschlussreich, da hier sprachliches und fachliches Lernen explizit als Unterrichtsziele vorgegeben sind. Die Ausführungen zur Interaktion zwischen Sprache und fachlichem Lernen sowie ein Exkurs zu bilingualen Lernkontexten erlaubt es abschließend sowohl spezifische Erklärungen von Leistungsdefiziten bei Kindern mit schwachen sprachlichen Kompetenzen als auch Grundlagen für eine allgemein förderliche Gestaltung von Unterricht abzuleiten.

\section{Empirische Befunde zur Funktion der Sprache beim fachlichen Lernen}

Tatsächlich ist der Zusammenhang von sprachlichen Kompetenzen und schulischem Erfolg vielfach belegt. Selbst unter Kontrolle relevanter Drittvariablen wie dem sozioökonomischen Status (SES), des kulturellen Kapitals oder den kognitiven Grundfähigkeiten erweist sich der Zusammenhang für Schulfächer wie Mathematik und Naturwissenschaften als substanziell (z. B. Fuchs et al., 2016; Kempert et al., 2016; Pöhlmann, Haag \& Stanat, 2013; Tarelli, Schwippert \& Stubbe, 2012). Die meisten Befunde stammen dabei aus korrelativen LargeScale-Studien, in denen sprachliche Kompetenz ausschließlich über Lesekompetenz operationalisiert wird. Ergänzt werden die Befunde durch einige längsschnittliche Untersuchungen, die den Zusammenhang von Sprache und schulischen Kompetenzen untermauern (z. B. Paetsch \& Felbrich, 2015; Paetsch, Felbrich \& Stanat, 2015). Über die Studien hinweg zeigen sich substanzielle Korrelationen zwischen sprachlichen Kompetenzen und fachlichem Lernen. Kinder mit schwachen sprachlichen Kompetenzen weisen dementsprechend auch schwache fachliche Leistungen auf. Allerdings geben die Studien aufgrund ihres korrelativen Charakters keinen direkten Aufschluss darüber, wie die hohen Zusammenhänge von sprachlichen und fachlichen Kompetenzen im Detail zu erklären sind.

Eine mögliche Interpretation der Zusammenhänge basiert auf der Annahme, dass bei schwachen sprachlichen Voraussetzungen die Probleme unmittelbar beim (Lese-)Verständnis der Testaufgaben entstehen (Haag, Heppt, Stanat, Kuhl \& Pant, 2013). Die in Large-ScaleStudien eingesetzten kompetenzorientierten Aufgaben sind meist sprachlich stark kontextualisiert und damit in ihrer Schwierigkeit möglicherweise sprachkompetenzabhängig. Erhöhte sprachliche Anforderungen durch die Kontextualisierung der Aufgaben könnten beispielsweise erklären, warum das Leseverständnis in der dritten Klasse die Mathematikleistung in 
der achten Klasse voraussagt (z. B. Grimm, 2008; siehe auch Fuchs et al., 2016). Insgesamt weist die Studienlage zur Rolle sprachlicher Merkmale bei Testaufgaben jedoch eher auf kleine Effekte hin (Kieffer, Lesaux, Rivera \& Francis, 2009; Kieffer, Rivera \& Francis, 2012).

Eine weitere Erklärung zielt auf den fachlichen Lehr-Lern-Prozess selbst ab. In diesem Szenario ist die Sprache das zentrale Medium der Vermittlung und der individuellen Wissenskonstruktion. Demnach führen schwache Sprachkompetenzen zu Defiziten sowohl im Verständnis zentraler Konzepte (also beim fachlichen Lernen) als auch bei prozessbezogenen Kompetenzen (z. B. beim Argumentieren), die sich dann in der Testsituation manifestieren (vgl. Paetsch et al., 2015; Prediger, Wilhelm, Büchter, Gürsoy \& Benholz, 2015). Diese Erklärung unterstützen Befunde von Kempert et al. (2011) sowie Saalbach et al. (2016). In diesen Studien war (mündliche) Sprachkompetenz neben arithmetischer Grundfertigkeit der stärkste Prädiktor für das erfolgreiche Bearbeiten von mathematischen Textaufgaben bei Grundschülerinnen und -schülern. Die Prädiktion war dabei tatsächlich stärker als diejenige von kognitiven Grundfähigkeiten, SES und Leseverständnis und zeigte sich sowohl bei Kindern mit als auch bei Kindern ohne Zuwanderungshintergrund. Diese Interpretation lässt sich im Rahmen konstruktivistischer Lerntheorien nachvollziehen, die der Sprache eine zentrale Rolle beim Wissenserwerb beimessen (Reusser, 2001; Schleppegrell, 2010). Im mathematischen oder naturwissenschaftlichen Unterricht kann beispielsweise die Verwendung komprimierter, dekontextualisierter Sprache als eine bestimmte fachspezifische Sprachform gesehen werden, die gleichzeitig Ausdruck und Voraussetzung für komplexes fachliches Lernen ist. Zusammenfassend nimmt die Sprache beim fachlichen Unterricht folglich sowohl eine kommunikative Funktion bei der Vermittlung von Informationen als auch eine kognitive Funktion bei der Konstruktion neuen Wissens ein (vgl. auch den Begriff der epistemischen Funktion der Sprache bei Morek \& Heller, 2012).

\section{Die kommunikative und die kognitive Funktion von Sprache beim fachlichen Lernen}

Nach den im vorherigen Abschnitt dargestellten empirischen Ergebnissen und deren Erklärungen können Schwächen in der Instruktionssprache sowohl zu kommunikativen als auch kognitiven Hürden führen und damit fachliches Lernen erschweren.

Kommunikative Hürden entstehen in erster Linie durch fehlende sprachliche Mittel, insbesondere durch fehlenden Alltagswortschatz (siehe z. B. Ahrenholz, 2010 für einen Überblick). Zusätzlich zum Alltagswortschatz muss in der Schule fachabhängig auch ein entsprechender Fachwortschatz aufgebaut bzw. eine Bedeutungsdifferenzierung alltäglicher Begriffe geleistet werden. Viele alltägliche Begriffe haben innerhalb des fachlichen Kontextes eine vom Alltagsgebrauch abweichende oder eine sehr spezifisch definierte Bedeutung (Pimm, 1987). Das Verständnis der Kommunikation im Unterricht oder das Leseverständnis ist somit in doppelter Hinsicht gefährdet. Erstens werden neue (Fach-)Wörter langsamer gelernt, wenn weniger Grundwortschatz vorhanden ist - denn damit fehlen Gelegenheiten, Analogien zu bilden oder Ableitungen vorzunehmen (Ahrenholz, 2010). Zweitens erfordert die Erschließung der Bedeutung neuer Begriffe oder die konzeptuelle Umstrukturierung bekannter Wörter beim fachlichen Lernen (z.B. der Begriffe Arbeit und Kraft in der Physik) Arbeitsgedächtnisressourcen (siehe Abschnitt zum Zusammenhang von sprachlicher und kognitiver Entwicklung im Kindesalter und dessen Auswirkungen auf den Wissenserwerb). Diese Ressourcen stehen dann nicht für schlussfolgerndes Denken, Problemlösungen oder weitere Elaboration des eigentlichen Lerngegenstands zur Verfügung (vgl. Daneman \& Green, 1986; van Dijk \& Kintsch, 1983). Ähnliche Mechanismen können nicht nur für Fachtermini, sondern auch für den Erwerb fachspezifischer Kollokationen (eine Summe bilden) oder Diskursformen (z. B. Argumentieren) angenommen werden. Es kann bis weit ins Jugendalter 
hinein dauern, bis ein Verständnis selbst von recht konkreten Wörtern erreicht wird, das dem von Erwachsenen ähnlich ist (Ameel, Malt \& Storms, 2008; Saji et al., 2011). Sogar bei fortgeschrittenen Physikstudierenden, die sich mit Quantenmechanik beschäftigen, konnte gezeigt werden, wie sie basierend auf grammatikalischen Strukturen und basalen Metaphern die Erklärungen ihres Dozierenden ontologisch falsch kategorisieren, Prinzipien übergeneralisieren und fehlerhaft anwenden (Brookes \& Etkina, 2007). Beispielsweise ist Energie physikalisch über eine Zustandsfunktion definiert, gesprochen wird aber häufig über Energiefluss und Ähnliches, da es kaum möglich ist, Zustandsfunktionen sprachlich zu kommunizieren - die Lernenden schlussfolgern fälschlicherweise, dass Energie Materie sei. Sind die sprachlichen Mittel zum Austausch über das Konzept eingeschränkt, wird ein fachbezogener Diskurs mit den Elementen Benennen, Beschreiben, Erklären und Argumentieren schwierig.

Die kognitive Funktion von Sprache wird deutlich, wenn die Kommunikationshürden den Aufbau und die Verwendung von Konzepten oder Prozeduren erschweren oder gar hemmen. Mit einem Konzept, für das noch kein sprachliches Repertoire und damit keine explizierbare kognitive Repräsentation vorhanden ist, sind kaum weitere kognitive Operationen möglich, die für Problemlösen, Schlussfolgerungen und Transfer notwendig wären, also für Fähigkeiten, die fachliche Kompetenz im engeren Sinne ausmachen. Man denke hier beispielsweise an das Konzept der Brüche in der Mathematik, bei dem zwei Zahlen als eine "gesprochen" werden - dies weicht stark von den zuvor erworbenen mathematischen Konzepten (Grundrechenarten) ab. Neu zu erwerbende Konzepte beim fachlichen Lernen werden immer wieder solche Herausforderungen stellen. Wenn ein angemessenes und flexibel nutzbares sprachliches Repertoire nicht vorhanden ist oder nicht im Unterricht entwickelt wird, bleibt Auswendiglernen der durch die Lehrperson gegebenen verbalen Beschreibung oder der in einem Text angeführten Definition die einzige Möglichkeit für Lernende.
Sprache stellt also nicht nur das Medium zum Verständnis eines konkreten Konzeptes dar, sondern bildet auch das Werkzeug, um mit diesem Konzept kognitiv zu operieren. Spezifische Aspekte der unterrichtlichen Sprachnutzung spielen dabei eine entscheidende Rolle: Wie gut man ein zunächst unbekanntes Konzept durch Analogiebildung (z. B. Kuchenanalogie für mathematische Brüche) erschließen kann, ist u. a. von der Verwendung und dem Verständnis relationaler Sprache abhängig. Die Nutzung generischer Sprache kann zudem - wie oben geschildert - die Tiefe der kognitiven Verarbeitung beeinflussen und damit die Qualität der möglichen Schlussfolgerungen und die Wissensorganisation (Gelman, Sánchez Tapia \& Leslie, 2016; Gelman, Ware \& Kleinberg, 2010).

Möglichkeiten, wie die kommunikative und kognitive Funktion von Sprache unter Rückgriff auf bestehende Konzepte und das Wissen um die Wirkweise spezifischer sprachlicher Konstruktionen auf die kognitive Verarbeitung berücksichtigt werden kann, werden im Abschnitt Sprachnutzung und sprachliche Unterstützung im Unterricht skizziert.

\section{Exkurs: Fachliches Lernen in bilingualen Kontexten}

Vor dem Hintergrund der Befunde zur kognitiven und kommunikativen Funktion von Sprache beim fachlichen Wissenserwerb ist die Frage naheliegend, inwieweit bilinguale Lehr-LernKontexte den Lernprozess beeinflussen. Ergebnisse aus Studien zum fachlichen Lernen in bilingualen Kontexten könnten einerseits darüber Aufschluss geben, wie fachliches Lernen unter expliziter Berücksichtigung der sprachlichen Voraussetzungen der Schülerinnen und Schüler organisiert werden kann, um gleichzeitig sprachliche und fachliche Kompetenzen zu entwickeln. Andererseits bieten diese Studien Erkenntnisse dazu, wie fachlicher Wissenserwerb bzw. Wissensabruf gelingen kann, wenn die Kompetenzen der Schülerinnen und Schüler in der Unterrichtssprache erwartbar schwach sind bzw. wenn sich Unterrichtssprache und Testsprache voneinander unterscheiden. 
Fremdsprachlicher Fachunterricht (z. B. in Form von Immersionsunterricht oder Content and Language Integrated Learning, CLIL) erfreut sich im Primar-, Sekundar- und Hochschulbereich zunehmend großer Beliebtheit. Mit diesem Unterrichtskonzept werden zwei Ziele verfolgt: Gleichzeitig sollen neue Fachinhalte erlernt und Fremdsprachenkenntnisse erworben werden. Fächer wie Mathematik, Biologie oder Geschichte werden entsprechend in einer Sprache unterrichtet, die für die (meisten) Lernenden nicht die Muttersprache ist. Derartige Programme tragen nachweislich (und wegen der zusätzlich zum reinen Fremdsprachenunterricht aufgewendeten Zeit wenig überraschend) zur Entwicklung der Kompetenz in der unterrichteten Fremdsprache bei (Lo \& Lo, 2014; Zaunbauer, Gebauer \& Möller, 2012). Für das fachliche Lernen sind die Befunde jedoch weniger eindeutig (z. B. Rumlich, 2016; Zaunbauer \& Möller, 2010) oder das fachliche Lernen wurde in den Analysen nicht angemessen berücksichtigt (z. B. Bruton, 2013; Dalton-Puffer, 2011; für einen Überblick zu Formen und Effekten bilingualen Lernens siehe Möller et al., 2018). Aktuelle Studien, die fachliches Lernen im Immersionsunterricht erfassen und es mit dem Lernen von Schülerinnen und Schülern vergleichen, die in ihrer Muttersprache unterrichtet werden, ergeben bislang kein klares Bild. Während in einigen Studien keine substanziellen Nachteile für das fachliche Lernen festgestellt werden konnten (Dallinger, Jonkmann, Hollm \& Fiege, 2016; Fleckenstein et al., 2017), zeigten sich in anderen Studien teilweise deutliche Nachteile (Lo \& Lo, 2014; Piesche, Jonkmann, Fiege \& Keßler, 2016; Roussel, Joulia, Tricot \& Sweller, 2017). Eine Erklärung für dieses unklare Bild könnte sein, dass Lehrkräfte ihren Unterricht in unterschiedlichem Ausmaß didaktisch sprachsensibel gestalten, um die sprachlichen Voraussetzungen ihrer Schülerschaft zu berücksichtigen (vgl. Baumert, Köller \& Lehmann, 2012). Manchen Lehrkräften gelingt es scheinbar, ihr fachliches Unterrichtsangebot an die sprachlichen Ausgangslagen ihrer Schülerinnen und Schüler anzupassen und/oder bei Verständnisproblemen diagnostisch zwischen fachlichen und sprachlichen Hürden zu unterscheiden sowie angemessene Unterstützungsangebote zu machen. Leider geht jedoch aus den Studien nicht hervor, wie die Interaktionen im Unterricht konkret gestaltet wurden. Daher können keine belastbaren Rückschlüsse über die Organisationsform und die allgemeinen Unterrichtsmethoden hinaus gezogen werden.

Eine mögliche Erklärung, insbesondere für nachteilige Effekte von Immersionsansätzen und CLIL, bieten experimentelle Laborstudien, die eine Gebundenheit des Wissens an die Lernsprache nahelegen (z. B. Marian \& Fausey, 2006; Gentner \& Goldin-Meadow, 2003; Spelke \& Tsivkin, 2001). Dies steht im Widerspruch zu den in Immersionsansätzen (implizit) getroffenen Annahmen, dass Wissen in einem kognitiven System unabhängig von der jeweiligen Instruktionssprache repräsentiert wird. Die Laborstudien geben deutliche Hinweise auf kognitive Kosten, die entstehen, wenn Wissen in einer Sprache abgerufen wird, die sich von der Instruktionssprache unterscheidet. Beim Lernen von mathematischen Inhalten konnten beispielsweise kognitive Kosten in Form von längeren Reaktionszeiten und höheren Fehlerzahlen nachgewiesen werden, wenn die Sprache beim Abruf von arithmetischem (Fakten-)Wissen nicht mit der Instruktionssprache übereinstimmt (Grabner et al., 2012; Hahn, Saalbach \& Grabner, 2017; Saalbach et al., 2013; Volmer, Grabner \& Saalbach, 2018).

Die genaue Bestimmung der Interaktionen zwischen Sprache und fachlichem Lernen in bilingualen Kontexten weist hier allerdings noch deutlichen Forschungsbedarf auf. Ergebnisse dieser Forschung, insbesondere hinsichtlich der Art der Umsetzung von fachlichem Unterricht in einer Fremdsprache, könnten zukünftig möglicherweise auch wertvolle Hinweise für den Umgang mit sprachlichen Herausforderungen im regulären Fachunterricht bieten. Wichtig für den vorliegenden Beitrag sind jedoch zwei zentrale Erkenntnisse der Forschung zum fachlichen Lernen in bilingualen 
Kontexten: erstens, dass die Repräsentation von fachlichem Wissen nicht unabhängig von ihrer sprachlichen Repräsentation ist, und zweitens, dass Lehrkräfte nicht nur das fachliche, sondern auch das fachsprachliche Lernen im Blick haben müssen. Sie müssen verfolgen, ob Lernschwierigkeiten aus mangelnder Sprachkompetenz oder mangelndem fachlichen Verständnis resultieren.

\section{Sprachnutzung und sprachliche Unterstützung im Unterricht}

Bei der Betrachtung des Zusammenhangs zwischen Sprache und Lernen entsteht häufig der Eindruck, Sprache sei die Voraussetzung für fachliches Lernen von Kindern. Sprachliche, kognitive sowie fachliche Fähigkeiten entwickeln sich jedoch, wie beschrieben, noch bis weit in die Adoleszenz (und darüber hinaus) interagierend, sodass die Wirkrichtung oft unklar ist (Ehlich et al., 2008; Gentner, 2016). Im (Fach-)Unterricht der Schule liegt der Fokus in erster Linie auf der Vermittlung fachlicher Inhalte, dabei wird eine angemessene sprachliche sowie kognitive Entwicklung meist einfach vorausgesetzt (Härtig, Bernholt, Prechtl \& Retelsdorf, 2015; Rincke, 2010; Snow, 2010). Neben allgemeinen Unterschieden in der sprachlichen Kompetenz zwischen den Kindern bleibt dann z. B. unberücksichtigt, dass die Begriffsdifferenzierung in vielen Fällen noch nicht abgeschlossen ist. Es ist ein langwieriger Prozess, bis Kinder ein mit Erwachsenen vergleichbares Verständnis von spezifischen Begriffen und Wörtern erlangen. Selbst bei konkreten Begriffen (z. B. Wörter für Gefäße oder Handlungen) dauert die Begriffsdifferenzierung oft bis ins frühe Jugendalter an (Ameel et al., 2008; Saji et al., 2011). Weitere Aspekte, wie beispielsweise die Erkenntnis, dass Sprache kontextgebunden ist und daher hochgradig flexibel verwendet werden kann, sind ebenfalls stark entwicklungsabhängig und bleiben selbst für die meisten Erwachsenen herausfordernd (man denke an die Begriffe Arbeit und Kraft in ihrer physikalischen oder alltagssprachlichen Verwendung oder die unterschiedliche Bedeutung des Begriffs Lösung im Mathematik- und Chemieunterricht). Konsequenterweise sollten also bei der Planung und Durchführung von Unterricht sowohl sprachliche Aspekte des fachlichen Inhalts als auch die sprachlichen Voraussetzungen der Schülerinnen und Schüler explizit berücksichtigt und adressiert werden.

\section{Berücksichtigung der Sprache bei der Planung von Unterricht}

Häufig trifft man neben dem Begriff der Fachsprache auf den Begriff der sogenannten Bildungssprache, wenn die sprachlichen Herausforderungen im Unterricht der Schule charakterisiert werden (vgl. z. B. Cummins, 2000; Schleppegrell, 2004). Das Konzept der Bildungssprache soll dem Umstand Rechnung tragen, dass Sprache je nach Verwendungszusammenhang unterschiedlich anspruchsvoll sein kann. Bildungssprache gilt als eigenes, insbesondere schulisch relevantes, sprachliches Register, das eine Reihe idiosynkratrischer schwierigkeitsgenerierender Merkmale aufweist. Dabei wird in der Regel auf einen reduzierten Kontext sowie auf komplexe lexikalische, grammatische und diskursive Merkmale verwiesen (z. B. Gogolin, 2009; Leseman, Scheele, Mayo \& Messer, 2007; Morek \& Heller, 2012). Allerdings steht eine eindeutige und operationalisierbare Definition von Bildungssprache noch aus; eine klare Abgrenzung zur sogenannten Alltagssprache ist nach wie vor schwierig (z. B. Berendes, Dragon, Weinert, Heppt \& Stanat, 2013; Härtig et al., 2015; Snow, 2010). Häufig wird bei der Beschreibung von Bildungssprache wenig darauf eingegangen, dass die Komplexität nicht zwingend exklusiver Teil der Sprache ist, sondern dass die Vermittlung zunehmend komplexer Konzepte (z. B. Kraft, Vektor, Evolution) im Unterricht die Verwendung anspruchsvoller oder sehr spezifischer Sprachstrukturen notwendig macht. Komplexe Sprache kann somit auch ein Indikator für eine kognitiv anspruchsvolle Phase des Konzepterwerbs darstellen. Anders ausgedrückt: Es sind auch die anspruchsvollen 
Konzepte im Unterricht, die ein entsprechendes sprachliches Repertoire erfordern (Fürstenau $\&$ Lange, 2011; Snow \& Uccelli, 2009). Breit generalisierbare Konzepte, wie etwa das der physikalischen Kraft, der Vektorrechnung oder der Evolutionstheorie, werden nicht erst durch die sprachliche Vermittlung anspruchsvoll. Sowohl die sprachlichen als auch die inhaltlich-kognitiven Anforderungen sind in diesen Fällen hoch und interagieren. Folglich gehört zur Planung und Durchführung von Unterricht nicht nur die Analyse von (bildungs-)sprachlichen, sondern auch von inhaltlichen Herausforderungen des fachlichen Unterrichts. Mögliche sprachliche, kognitive oder inhaltliche Hürden für das fachliche Lernen sind genau einzuschätzen und in Bezug zu den Voraussetzungen der Schülerinnen und Schüler angemessen zu berücksichtigen.

\section{Fachlicher Unterricht als Anwendung sprachbewussten Handelns}

Losgelöst von integrierten Verfahren des sprachlichen und fachlichen Lernens, wie es in Immersionsansätzen oder beim CLIL realisiert wird (z. B. Möller et al., 2018), sowie spezifischen sprachlichen Trainingsprogrammen, die die kompensatorische Förderung sprachlicher Kompetenzen zum Ziel haben (siehe Überblick in Kempert et al., 2016), bietet der reguläre fachbezogene Unterricht der Schule ebenfalls verschiedene Möglichkeiten, sprachliche Unterstützung zu geben. Erstens sollten allgemeine und fachsprachliche bzw. bereichsspezifische sprachliche Kompetenzen gefördert werden. Zweitens sollte durch ein sprachbewusstes Vorgehen das fachliche Lernen einer sprachlich heterogenen Schülerschaft gesichert und unter Berücksichtigung der oben geschilderten $\mathrm{Zu}$ sammenhänge von Sprachnutzung und kognitiver Verarbeitung unterstützt werden.

Dabei ist die Berücksichtigung sprachlicher Facetten im Fachunterricht nicht nur unter dem Gesichtspunkt der Vermeidung kommunikativer und kognitiver Hürden zu sehen. Vielmehr bietet der Fachunterricht für bestimmte sprachliche Konstruktionen überhaupt erst Anwendungsgelegenheiten oder sogar besonders motivierende Erwerbssituationen. Als Beispiel dafür kann das naturwissenschaftliche Experiment herangezogen werden, welches in besonderer Weise Handlung, Sprechen und Denken verzahnt (Kuhn, Iordanou, Pease \& Wirkala, 2008). In diesem Kontext können beispielsweise Vermutungen gesammelt werden und damit sprachliche Formen des Möglichen bzw. Zukünftigen erarbeitet werden („Ich denke, es wird ...", „Ich vermute, dass ..."). Ebenso ist in diesem Zusammenhang häufig die Nutzung verschiedener Formen von Konditionalsätzen notwendig - Lernende können so deren grammatische und fachliche Besonderheiten kennenlernen („Je ..., desto ...", „Wenn ..., dann ..."). Ferner können durch die Exploration und den Vergleich von Materialien sowie deren Eigenschaften auch ungewöhnliche Begriffe oder graduelle Differenzen zwischen Begriffen angesprochen und eingeübt werden (z. B. rau, porös, spröde; „Das hier ist schwerer als ..."). Bei der konkreten Durchführung eines Experiments können durch die sprachliche Begleitung (Parallelsprechen) zahlreiche Handlungen und $\mathrm{Ab}$ läufe expliziert werden, die durch die direkte Sinneserfahrung und zusätzliche Visualisierung eine einprägsame Erwerbssituation darstellen. Die Interpretation und Kommunikation der Ergebnisse eines Experiments bilden weitere wertvolle Gelegenheiten, um Diskurs- und Argumentationsformen zu modellieren und üben zu lassen.

Für die allgemeine fachübergreifende Beschreibung solcher und weiterer Maßnahmen der integrierten sprachlichen und fachlichen Förderung stellt der Begriff des sogenannten Scaffolding einen sinnvollen konzeptuellen Rahmen für sprachbewusstes Handeln dar (Mannel, Hardy, Sauer \& Saalbach, 2016; siehe auch Hardy et al., in diesem Heft) und wird daher im Folgenden näher beschrieben. Ergänzt wird diese Beschreibung durch Implikationen, die sich aus den obigen Ausführungen zum $\mathrm{Zu}$ sammenhang von Sprachnutzung, kognitiver Verarbeitung und Wissensaneignung ergeben. 


\section{Scaffolding als konzeptueller Rahmen für sprachliche und kognitive Unterstützung beim fachlichen Lernen}

Scaffolding ist zunächst nicht auf sprachliche Unterstützung beschränkt, sondern beschreibt individuelle, adaptive Unterstützungsmaßnahmen durch eine Lehrkraft im Unterricht zur Lösung von Aufgaben, die den Entwicklungsstand eines Lernenden gerade noch übersteigen (vgl. Hardy, 2012; van de Pol, Volman \& Beishuizen, 2010).

Bezogen auf sprachliches Scaffolding identifiziert Gibbons (2002) die Notwendigkeit der sprachlichen Bedarfsanalyse für den konkreten Unterrichtsinhalt. Diese Analyse ist abzugleichen mit dem sprachlichen und fachlichen Niveau der Lernenden. Ausgehend von dieser integrativen Betrachtung findet die Unterrichtsplanung statt, die dementsprechend die Verknüpfung von sprachlichen und fachlichen Aspekten beinhalten sollte. Diese drei Schritte werden unter dem Begriff des Makro-Scaffolding zusammengefasst. Letztlich sollte aus diesem Vorgehen und unter Rückgriff auf spezifische Techniken im Unterricht eine effiziente Lehr-Lern-Interaktion resultieren (das sog. Mikro-Scaffolding).

Beispiele für konkrete Mikro-ScaffoldingTechniken im Unterricht sind die Modellierung, die Fokussierung sowie die Korrektur (Gibbons, 2002). Unter Modellierung versteht man das sprachliche Verhalten der Lehrkraft. Äußerungen sollten sprachlich vorbildlich sein, dabei aber nicht grundsätzlich schwierige sprachliche Konstruktionen und Fachsprache vermeiden. Das Verständnis sollte eher durch die Regulation des Tempos während der Interaktionen zwischen Lehrenden und Lernenden sichergestellt werden. Verständnisprobleme der Schülerinnen und Schüler können zudem explizit im Unterrichtsgespräch aufgegriffen werden, um etwa die Begriffsdifferenzierung zu thematisieren. Dem Konzept des Scaffoldings folgend sollten sich die sprachlichen Anforderungen leicht über dem Niveau der Lernenden bewegen. So können Ausführungen durch Erklären von (Fach-)Begriffen, Bereitstellen von Analogien und sprachlichen Varianten angereichert werden.
Das (sprachliche) Vorwissen wird somit berücksichtigt und erweitert, ohne dabei kognitive Ressourcen wie das Arbeitsgedächtnis zu überlasten. Die Wirksamkeit der sprachlichen Modellierung ist empirisch belegt. Befunde zeigen, dass die Komplexität der verwendeten Sprache von Modellen, wie den Eltern oder den Lehrkräften, einen klaren Zusammenhang mit der sprachlichen und kognitiven Entwicklung von Kindern beispielsweise im Bereich der Syntaxentwicklung, aber auch beim mathematischen Verständnis aufweisen (Huttenlocher, Vasilyeva, Cymerman \& Levine, 2002; Klibanoff, Levine, Huttenlocher, Vasilyeva \& Hedges, 2006; Mascareño, Snow, Deunk \& Bosker, 2016).

Die Sicherung und Bereitstellung des jeweils relevanten Wortschatzes oder fachspezifischer Kollokationen durch Modellierung ist hierbei nicht nur unter der Prämisse der kommunikativen Funktion von Sprache bedeutsam. Für diese Funktion gibt es, neben den genannten, zahlreiche weitere Möglichkeiten, die von Wortschatzlisten über Karteikartensysteme bis hin zu grafisch aufbereiteten Mindmaps reichen. Um zudem Schülerinnen und Schülern auch die selbstständige sprachliche Elaboration zu ermöglichen und damit die aktive Wissenskonstruktion zu unterstützen (wie im Abschnitt Der Zusammenhang von sprachlicher und kognitiver Entwicklung im Kindesalter und dessen Auswirkungen auf den Wissenserwerb dargelegt), sollten neben dem passenden Wortschatz auch entsprechende Strategien vermittelt sowie deren Nutzung angeregt werden (vgl. Renkl, 2009). Dazu gehören u. a. das Auffordern zur Umformulierung von Erklärungen, das Generieren von eigenen Beispielen und das Erstellen von Zusammenfassungen.

Sprachliche Herausforderungen auf Wortund Satzebene können durch die Technik der Fokussierung (i.S. von Aufmerksamkeitslenkung) adressiert werden. Zu diesen Herausforderungen gehören etwa Passivformen (z. B. wird berechnet), logische Bedingungssätze (unter Verwendung etwa von je/desto, wenn/dann) und Verknüpfungen (z. B. durch jedoch oder sodass). Ferner stellen Fachbegriffe (z. B. Fotosynthese), 
spezifische oder ungebräuchliche Wörter (z. B. Flaschenzug) und solche mit verschiedenen Bedeutungen (z. B. Lösung), Nominalisierungen (z.B. die Unbekannte), Komposita (z. B. Versuchsaufbau) und reflexive Verben (z. B. sich rückbeziehen) besondere Hürden dar. Durch die explizite Lenkung der Aufmerksamkeit auf eben diese Hürden können Unklarheiten gezielt aufgegriffen werden.

Wie beschrieben ist die Rolle der Sprache für die Bildung von Kategorien, das schlussfolgernde Denken sowie den Transfer von Gelerntem und damit für unterrichtliches Handeln über alle Fächer hinweg relevant. Scaffolding-Maßnahmen sind auch hier wirksam und lassen sich ebenfalls unter dem Begriff der Fokussierung beschreiben. Gerade im fachlichen Unterricht, in dem mit verschiedenen Materialien und Repräsentationen (z. B. mit Texten, Grafiken, hands-on-Materialien) integrativ umgegangen werden muss, ist Fokussierung essenziell. Zum Beispiel kann im Unterricht, in dem das Thema der physikalischen Dichte anhand des Schwimmens und Sinkens von Gegenständen konkretisiert wird, durch die explizite Benennung des verwendeten Materials (wie etwa Eisen, Blei, Aluminium [Metall]; Brett, Platte, Klotz [Holz]) und seines Verhaltens im Wasser (schwimmen, schweben, sinken, tauchen, gleiten, treiben) die Begriffsdifferenzierung und Kategorienbildung stimuliert werden (Leuchter \& Saalbach, 2014). Durch den reflektierten Einsatz von spezifischen („Schau mal, dieser Klotz schwimmt!“, „Schau mal, der Klotz aus Holz schwimmt!") und von generischen Äußerungen („Schau mal, Holz schwimmt!") kann zudem die Tiefe der kognitiven Verarbeitung beeinflusst werden, was sich dann in einer verbesserten Transferleistung zeigt (vgl. Gelman et al., 2009). Ferner ist der gezielte Einsatz von relationaler Sprache zur Fokussierung geeignet. Relationale Sprache lenkt, wie zu Beginn dargestellt, die Aufmerksamkeit („Schaut, ich habe zwei gleich große Würfel: einen aus Holz, einen aus Metall. Mal sehen, welcher schwimmt und welcher sinkt"), unterstützt die Bildung von Analogien („Nun vergleicht mal ein anderes Paar von Materialien auf die gleiche Weise") und fördert auf diese Prozesse aufbauendes schlussfolgerndes Denken („Wir haben jetzt viele Würfel aus verschiedenen Materialien verglichen. Was hat einen Einfluss darauf, dass etwas schwimmt oder sinkt?"; vgl. Gentner, 2010; Loewenstein \& Gentner, 1998).

Äußerungen der Schülerinnen und Schüler, die sich noch auf einem (zu) einfachen Niveau befinden oder fehlerhaft sind, können durch die Technik der Korrektur lernwirksam genutzt werden. Bei der Korrektur oder dem korrektiven Feedback wird beispielsweise die Reformulierung einer zunächst sprachlich fehlerhaften Äußerung angeboten (hier zählt also wieder die Modellierung durch die Lehrperson), ohne dass direkt auf die Fehler eingegangen wird. Zudem können bei diesem Vorgehen Äußerungen elaboriert und durch Fachwortschatz bzw. fachtypische Formulierungen angereichert und somit indirekt bereitgestellt werden (z. B. „Das geht bestimmt unter!“ umformulieren in „Du vermutest also, dass der Würfel aus Metall sinkt?"). Umgekehrt können auch die Lernenden aufgefordert werden, eine Arbeitsanweisung oder Aufgabenstellung in eigene Worte zu fassen, und damit zu längeren, fachlichen Äußerungen ermutigt werden. Damit sichert man zum einen das Verständnis des inhaltlichen Auftrags, zum anderen bietet man Gelegenheit, das Verständnis und die Produktion von Fachsprache einzuüben.

Die genannten Techniken stellen hohe Anforderungen an die Lehrkraft im Fachunterricht. Zusätzlich zum fachlichen und fachdidaktischen Wissen ist ein hochgradig kontrolliertes und reflektiertes Sprachverhalten gefordert, das auch das Bewusstsein für sprachliche Hürden der Fachsprache umfasst (siehe auch Leuchter \& Saalbach, 2014). Lehrkräfte müssen Möglichkeiten kennen, wie sie diese Hürden im Unterricht effektiv bearbeiten können. In Ergänzung zum flexiblen Umgang mit den genannten Mikro-Scaffolding-Techniken des Modellierens, Fokussierens und der Korrektur ist auch diagnostische Kompetenz zur Feststellung aktueller Sprachstände und der inhaltlichen Anforderung des Fachinhalts notwendig 
(Makro-Scaffolding). Nur so wird es möglich, bei der Unterrichtsplanung und Unterrichtsdurchführung eine Justierung zwischen dem $\mathrm{Ni}$ veau der Unterrichtssprache und dem Sprachstand der Lernenden vornehmen zu können. Kommt noch eine ausgeprägt heterogene Schülerschaft hinzu, muss die Unterrichtsgestaltung auch diesem Umstand Rechnung tragen und Maßnahmen müssen noch differenzierter und adaptiver angeboten werden. Um diese adaptive Unterstützung bieten zu können, müssen die vorgeschlagenen generellen und fächerübergreifenden Scaffolding-Ansätze durch zusätzliche spezifische fachdidaktische Maßnahmen an spezifische Inhalte angepasst und konkretisiert werden - hier liegt ein wichtiges Feld zukünftiger fachdidaktischer Forschung.

\section{Fazit und Ausblick}

Anhand der geschilderten Befunde lässt sich deutlich die wechselseitige Abhängigkeit der sprachlichen und der kognitiven Entwicklung im Kindesalter nachzeichnen. Sprache hat sowohl in ihrer kommunikativen als auch in ihrer kognitiven Funktion einen erheblichen Einfluss auf den Erfolg fachlichen Lernens. Beide Funktionen sind bedeutsam für die Vermittlung fachlicher Kompetenzen, die Konstruktion individuellen Wissens sowie den Abruf und Transfer des Gelernten. Wenn diese Funktionen nicht in ausreichendem Maße in der Vorbereitung und Durchführung von Unterricht berücksichtigt werden, ist das fachliche Lernen insbesondere bei sprachlich heterogenen Lerngruppen gefährdet. Gleichzeitig aber bieten gerade fachliche Lehr-Lern-Situationen vielfältige Möglichkeiten, die sprachlichen Kompetenzen der Lernenden durch sprachliches und kognitives Scaffolding zu entwickeln bzw. sprachliche Schwächen gezielt aufzugreifen. Die Bedeutung der Sprache bzw. die Stärke der Interaktion zwischen sprachlicher, kognitiver und fachlicher Entwicklung ist sicherlich auch fachabhängig, und Überlegungen zum jeweiligen Unterricht müssen demnach fachdidaktisch ausformuliert werden. Grundsätzliches
Wissen, z. B. über die Wirkung von generischer oder relationaler Sprache, den Nutzen von Strategien des sprachlichen Elaborierens etc., ist jedoch fächerübergreifend bedeutsam, ebenso wie das Konzept des (sprachlichen) Scaffoldings als wichtige Analyse-, Planungs- und Handlungsgrundlage für Lehrkräfte.

In der fachdidaktischen Forschung wird zunehmend darauf hingewiesen, dass das erfolgreiche Lernen innerhalb einer Disziplin ebenfalls ein gutes Verständnis der spezifischen Fachsprache sowie eine angemessene Nutzung dieser Fachsprache erfordert (Brookes \& Etkina, 2007; Rincke, 2011; Roth \& Duit, 2003). So betont Schleppegrell (2007): „Learning the language of a new discipline is a part of learning the new discipline" (S. 140). Ebenso gibt es eine ganze Reihe von Forschungsansätzen zu alltags- bzw. fachintegrierter Sprachförderung und zu immersiven Unterrichtsformaten, die relevante Erkenntnisse zum Verhältnis von fachlichem Lernen und Sprache bereithalten (Henschel, Stanat, Becker-Mrotzek, Hasselhorn \& Roth, 2014; Lütke, Petersen \& Tajmel, 2017; Möller et al., 2018). Jedoch wird die komplexe Interaktion von Sprache, kognitiven Prozessen und fachlichem Lernen in der empirischen fachdidaktischen oder sprachwissenschaftlichen Forschung noch zu selten angemessen berücksichtigt (vgl. Härtig et al., 2015), kaum in fachdidaktische Konzepte integriert und nur in wenigen Fällen in der Entwicklung von Unterrichtsmaterialien mitgedacht.

Neben der stärkeren Verankerung des Themas in der Forschung und Entwicklung sollten die Erkenntnisse zur Interaktion von Sprache, Kognition und fachlichem Lernen ein fester Bestandteil in der Aus- und Weiterbildung von Lehrkräften sein. Wissen über diese Interaktion kann als Teil professioneller Kompetenz dem fachdidaktischen und dem pädagogisch-psychologischen Wissen zugeordnet werden (vgl. Baumert \& Kunter, 2006). Teilweise findet der Einbezug sprachlicher Aspekte in der fachlichen Professionalisierung von Lehrkräften auch schon statt (vgl. KMK, 2004; oder KMK, 2008). Allerdings unterscheidet sich die konkrete Ausge- 
staltung zwischen den Bundesländern stark und es besteht weiterhin hoher Qualifizierungsbedarf (Baumann \& Becker-Mrotzek, 2014; MorrisLange, Wagner \& Altinay, 2016).

Bei der Betrachtung von Sprache, Denken und fachlichem Lernen darf nicht der Eindruck entstehen, dass sich diese klar voneinander trennen lassen. Ein instruktionaler Auftrag oder eine fachliche Erklärung ist ohne die Nutzung von Sprache praktisch nicht umsetzbar; gleiches gilt für die Bearbeitung einer Aufgabe durch Lernende. Bestimmte linguistische Konstruktionen und Konventionen müssen innerhalb eines Bereichs oder einer Disziplin kennengelernt und erworben werden (wobei es dazu auch noch relativ große Unterschiede zwischen Disziplinen wie beispielsweise Natur- und Geisteswissenschaften gibt; siehe Medimorec, Pavlik, Olney, Graesser \& Risko, 2015). Erst dann werden bestimmte Argumentationen für Lernende nachvollziehbar, erst dann können Lernende ihr Wissen ausdrücken und in Verhalten umsetzen und erst dann kann man von fachlicher Kompetenz sprechen (Deiglmayr, Schalk \& Stern, 2017; Lupyan, 2012).

Die pädagogisch-psychologische und fachdidaktische Forschung hat begonnen, sich mit der wechselseitigen Beeinflussung von Sprache, Kognition und fachlichem Lernen zu beschäftigen. Der vorliegende Beitrag betont durch die Zusammenführung unterschiedlicher empirischer Forschungsbefunde, wie zentral diese Interaktion für die Wirkung von Erziehung und Unterricht ist. Der Überblick ermöglicht es zudem, generelle, fachübergreifende Vorschläge für die praktische Unterrichtsplanung und -durchführung abzuleiten. Indem die behandelten Wechselwirkungen im Kontext von Lehren und Lernen vermehrt zum Gegenstand der Forschung und der Aus- und Weiterbildungen gemacht werden, kann perspektivisch die Gestaltung von Erziehung und Unterricht weiterentwickelt werden. Die reflektierte und angemessene Verwendung von Sprache beim fachlichen Lernen kann letztlich als Aspekt konstruktiver Unterstützung und damit als genuines Merkmal von Unterrichtsqualität verstanden werden.

\section{Literatur}

Ahrenholz, B. (2010). Bildungssprache im Sachunterricht in der Grundschule. In B. Ahrenholz (Hrsg.), Fachunterricht und Deutsch als Zweitsprache (S. 15-35). Tübingen: Narr.

Alderson-Day, B. \& Fernyhough, C. (2015). Inner speech: Development, cognitive functions, phenomenology, and neurobiology. Psychological Bulletin, 141, 931-965. https://dx.doi.org/10.1037/bul0000021

Ameel, E., Malt, B. \& Storms, G. (2008). Object naming and later lexical development: From baby bottle to beer bottle. Journal of Memory and Language, 58, 262-285. https://dx.doi.org/10.1016/j.jml.2007.01.006

Baumann, B. \& Becker-Mrotzek, M. (2014). Sprachförderung und Deutsch als Zweitsprache an deutschen Schulen: Was leistet die Lehrerbildung? Überblick, Analysen und Handlungsempfehlungen. Köln: Mercator-Institut für Sprachförderung und Deutsch als Zweitsprache.

Baumert, J., Köller, O. \& Lehmann, R. H. (2012). Leseverständnis im Englischen und Deutschen und Mathematikleistungen bilingual unterrichteter Schülerinnen und Schüler am Ende der Grundschulzeit. Ergebnisse eines Zwei-Wege-Immersionsprogramms. Unterrichtswissenschaft, 40, 290-315.

Baumert, J. \& Kunter, M. (2006). Stichwort: Professionelle Kompetenz von Lehrkräften. Zeitschrift für Erziehungswissenschaft, 9, 469-520. https://dx.doi.org/10.1007/ s11618-006-0165-2

Berendes, K., Dragon, N., Weinert, S., Heppt, B. \& Stanat, P. (2013). Hürde Bildungssprache? Eine Annäherung an das Konzept „Bildungssprache“ unter Einbezug aktueller empirischer Forschungsergebnisse. In A. Redder \& S. Weinert (Hrsg.), Sprachförderung und Sprachdiagnostik: Interdisziplinäre Perspektiven (S. 17-41). Münster: Waxmann.

Brookes, D. T. \& Etkina, E. (2007). Using conceptual metaphor and functional grammar to explore how language used in physics affects student learning. Physical Review Special Topics - Physics Education Research, 3, 010105. https://dx.doi.org/10.1103/PhysRevSTPER.3.010 105

Bruton, A. (2013). CLIL: Some of the reasons why .... and why not. System, 41, 587-597. https://dx.doi.org/10. 1016/j.system.2013.07.001

Cimpian, A. \& Markman, E. M. (2008). Preschool children's use of cues to generic meaning. Cognition, 107, 19-53. https://dx.doi.org/10.1016/j.cognition.2007. 07.008

Cummins, J. (2000). Language, power, and pedagogy: Bilingual children in the crossfire (Bilingual education and bilingualism: Vol. 23). Clevedon: Multilingual Matters. https://dx.doi.org/10.21832/9781853596773

Dallinger, S., Jonkmann, K., Hollm, J. \& Fiege, C. (2016). The effect of content and language integrated learning on students' English and history competences - Killing two birds with one stone? Learning and Instruction, 41, 23-31. https://dx.doi.org/10.1016/j.learninstruc.20 15.09 .003

Dalton-Puffer, C. (2011). Content-and-language integrated learning: From practice to principles? Annual Review of Applied Linguistics, 31, 182-204. https://dx. doi.org/10.1017/S0267190511000092

Daneman, M. \& Green, I. (1986). Individual differences in comprehending and producing words in context. Journal of Memory and Language, 25, 1-18. https:// dx.doi.org/10.1016/0749-596X(86)90018-5 
Dehaene, S. (1997). The number sense: How the mind creates mathematics. New York, NY: Oxford University Press.

Dehaene, S., Spelke, E., Pinel, P., Stranescu, R. \& Tsivkin, S. (1999). Sources of mathematical thinking: Behavioural and brain-imaging evidence. Science, 284 970 -974. https://dx.doi.org/10.1126/science.284.54 16.970

Deiglmayr, A., Schalk, L. \& Stern, E. (2017). Begabung, Intelligenz, Talent, Wissen, Kompetenz und Expertise: Einige Begriffsklärungen. In U. Trautwein \& M. Hasselhorn (Hrsg.), Begabungen und Talente: Tests und Trends - Jahrbuch der pädagogisch-psychologischen Diagnostik (Bd. 15, S. 1-16). Göttingen: Hogrefe.

Ehlich, K., Bredel, U. \& Reich H. H. (2008). Referenzrahmen zur altersspezifischen Sprachaneignung - Forschungsgrundlagen. Zugriff am 23. 8. 2018 unter https://www. bmbf.de/pub/Bildungsforschung_Band_29_2.pdf

Fernyhough, C. \& Fradley, E. (2005). Private speech on an executive task: Relations with task difficulty and task performance. Cognitive Development, 20, 103-120. https://dx.doi.org/10.1016/j.cogdev.2004.11.002

Fleckenstein, J., Möller, J., Hohenstein, F., Radmann, S., Becker, M. \& Baumert, J. (2017). Die schulischen Leistungen an der SESB: 9. Jahrgangsstufe und 15-Jährige. In J. Möller, F. Hohenstein, J. Fleckenstein, J. Baumert \& O. Köller (Hrsg.), Erfolgreich integrieren: Die staat liche Europa-Schule Berlin (S. 189-252). Münster: Waxmann.

Fuchs, L. S., Gilbert, J. K., Powell, S. R., Cirino, P.T., Fuchs, D., Hamlett, C. L., ... Tolar, T. D. (2016). The role of cognitive processes, foundational math skill, and cal culation accuracy and fluency in word-problem solving versus prealgebraic knowledge. Developmental Psychology, 52, 2085-2098. https://dx.doi.org/10.1037/ $\operatorname{dev} 0000227$

Fürstenau, S. \& Lange, I. (2011). Schulerfolg und sprachliche Bildung. Perspektiven für eine Unterrichtsstudie. In P. Hüttis-Graf \& P. Wieler (Hrsg.), Übergänge zwischen Mündlichkeit und Schriftlichkeit im Vor- und Grundschulalter (S. 37-54). Freiburg im Breisgau: Fillibach.

Gathercole, S.E. \& Baddeley, A. D. (1993). Working memory and language. Hillsdale, NY: Erlbaum.

Gathercole, S. E., Willis, C.S., Emslie, H. \& Baddeley, A. D. (1992). Phonological memory and vocabulary development during the early school years: A longitudinal study. Developmental Psychology, 28, 887-898. https://dx.doi.org/10.1037/0012-1649.28.5.887

Gelman, S.A. \& Markman, E. M. (1986). Categories and induction in young children. Cognition, 23, 183-209. https://dx.doi.org/10.1016/0010-0277(86)90034-X

Gelman, S.A., Raman, L. \& Gentner, D. (2009). Effects of language and similarity on comparison processing. Language Learning and Development, 5, 147 - 171. https:// dx.doi.org/10.1080/15475440902824079

Gelman, S.A., Sánchez Tapia, I. \& Leslie, S.-J. (2016). Memory for generic and quantified sentences in Spanish-speaking children and adults. Journal of Child Language, 43, 1231 - 1244. https://dx.doi.org/10.10 17/S0305000915000483

Gelman, S. A., Star, J. R. \& Flukes, J. (2002). Children's use of generics in inductive inferences. Journal of Cognition and Development, 3, 179-199. https://dx.doi. org/10.1207/S15327647JCD0302_3

Gelman, S.A., Ware, E.A. \& Kleinberg, F. (2010). Effects of generic language on category content and structure.
Cognitive Psychology, 61, 273-301. https://dx.doi.org/ 10.1016/j.cogpsych.2010.06.001

Gelman, S.A., Ware, E.A., Manczak, E. M. \& Graham, S.A (2013). Children's sensitivity to the knowledge expressed in pedagogical and nonpedagogical contexts. Developmental Psychology, 49, 491-504. https://dx. doi.org/10.1037/a0027901

Gentner, D. (2003). Why we're so smart. In D. Gentner \& S. Goldin-Meadow (Eds.), Language in mind: Advances in the study of language and thought (pp. 195-235). Cambridge, MA: MIT press.

Gentner, D. (2010). Bootstrapping the mind: Analogical processes and symbol systems. Cognitive Science, 34 752-775. https://dx.doi.org/10.1111/j.1551-6709. 2010.01114.x

Gentner, D. (2016). Language as cognitive tool kit: How language supports relational thought. American psychologist, 71, 650-657. https://dx.doi.org/10.1037/amp 0000082

Gentner, D. \& Goldin-Meadow, S. (Eds.) (2003). Language in mind: Advances in the study of language and thought. Cambridge, MA: MIT.

Gentner, D. \& Hoyos, C. (2017). Analogy and abstraction. Topics in Cognitive Science, 9, 672-693. https://dx.doi. org/10.1111/tops. 12278

Gibbons, P. (2002). Scaffolding language, scaffolding learning: Teaching second language learners in the main stream classroom. Portsmouth, NH: Heinemann.

Gogolin, I. (2009). Zweisprachigkeit und die Entwicklung bildungssprachlicher Fähigkeiten. In I. Gogolin \& U. Neumann (Hrsg.), Streitfall Zweisprachigkeit - The Bilingualism Controversy (S. 263-280). Wiesbaden: Springer. https://dx.doi.org/10.1007/978-3-531-915 96-8_15

Gordon, P. (2004). Numerical cognition without words: Evidence from Amazonia. Science, 306, 496-499. https:// dx.doi.org/10.1126/science.1094492

Grabner, R. H., Saalbach, H. \& Eckstein, D. (2012). Language-switching costs in bilingual mathematics learning. Mind, Brain, and Education, 6, 147-155. https:// dx.doi.org/10.1111/j.1751-228X.2012.01150.x

Grimm, K. J. (2008). Longitudinal associations between reading and mathematics achievement. Developmental neuropsychology, 33, 410-426. https://dx.doi.org/10. 1080/87565640801982486

Gunzenhauser, C., Hauch, F., Stephan, F. \& Saalbach, H. (in press). Verbal mediation planning in elemantary students: A think aloud approach.

Haag, N., Heppt, B., Stanat, P., Kuhl, P. \& Pant, H. A. (2013). Second language learners' performance in mathematics: Disentangling the effects of academic language features. Learning and Instruction, 28, 24-34. https://dx.doi.org/10.1016/j.learninstruc.2013.04. 001

Hahn, C. G. K., Saalbach, H. \& Grabner, R. H. (2017). Language-dependent knowledge acquisition: Investigating bilingual arithmetic learning. Bilingualism: Language and Cognition, 2, 1-11. https://dx.doi.org/10.1017/ S1366728917000530

Hardy, I. (2012). Kognitive Strukturierung in der Grundschule: Empirische Zugänge zu einem heterogenen Konstrukt der Unterrichtsforschung. In F. Hellmich, S. Förster \& F. Hoya (Hrsg.), Bedingungen des Lehrens und Lernens in der Grundschule: Bilanz und Perspektiven (S. 51-62). Wiesbaden: Verlag der Sozialwissenschaften. https://dx.doi.org/10.1007/978-3-531-19137-9_4 
Härtig, H., Bernholt, S., Prechtl, H. \& Retelsdorf, J. (2015). Unterrichtssprache im Fachunterricht - Stand der Forschung und Forschungsperspektiven am Beispiel des Textverständnisses. Zeitschrift für Didaktik der Naturwissenschaften, 21, 55-67. https://dx.doi.org/ $10.1007 /$ s40573-015-0027-7

Henschel, S., Stanat, P., Becker-Mrotzek, M., Hasselhorn, M. \& Roth, H.-J. (2014). Evaluationskonzept der BundLänder-Initiative „Bildung durch Sprache und Schrift“. Zugriff am 23. 8. 2018 unter http://www.biss-sprach bildung.de/pdf/Evaluationskonzept.pdf

Hirsh-Pasek, K. \& Golinkoff, R. M. (1999). The origins of grammar: Evidence from early language comprehension. Cambridge, MA: MIT.

Huttenlocher, J., Vasilyeva, M., Cymerman, E. \& Levine, S. (2002). Language input and child syntax. Cognitive Psychology, 45, 337-374. https://dx.doi.org/10.1016/ S0010-0285(02)00500-5

Imai, M. \& Saalbach, H. (2007, August). The role of labels on children's inductive reasoning. Paper presented at the 13th European Conference on Developmental Psychology, Jena, Germany.

Imai, M., Saalbach, H. \& Stern, E. (2010). Are chinese and german children taxonomic, thematic, or shape biased? Influence of classifiers and cultural contexts. Frontiers in Psychology, 1, 1-10. https://dx.doi.org/10.3389/fp syg.2010.00194

Imai, M., Schalk, L., Saalbach, H. \& Okada, H. (2014). All giraffes have female-specific properties: Influence of grammatical gender on deductive reasoning about sex-specific properties in German speakers. Cognitive Science, 38, 514-536. https://dx.doi.org/10.1111/ cogs. 12074

Kempert, S., Edele, A., Rauch, D., Wolf, K. M., Paetsch, J., Darsow, A., ... Stanat, P. (2016). Die Rolle der Sprache für zuwanderungsbezogene Ungleichheiten im Bildungserfolg. In C. Diehl, C. Hunkler \& C. Kristen (Hrsg.), Ethnische Ungleichheiten im Bildungsverlauf (S. 157-241). Wiesbaden: Springer. https://dx.doi. org/10.1007/978-3-658-04322-3_5

Kempert, S., Saalbach, H. \& Hardy, I. (2011). Cognitive benefits and costs of bilingualism in elementary schoo students: The case of mathematical word problems. Jour nal of Educational Psychology, 103, 547-561. https:// dx.doi.org/10.1037/a0023619

Kieffer, M. J., Lesaux, N.K., Rivera, M. \& Francis, D. J (2009). Accommodations for English language learners taking large-sale assessments: A meta-analysis on effectiveness and validity. Review of Educational Research, 79, 1168-1201. https://dx.doi.org/10.3102/00 34654309332490

Kieffer, M. J., Rivera, M. \& Francis, D. J. (2012). Practical guidelines for the education of English language learners: Research-based recommendations for the use of accommodations in large-scale assessments. 2012 update. Portsmouth, NH: RMC Research Corporation Center on Instruction.

Klibanoff, R. S., Levine, S.C., Huttenlocher, J., Vasilyeva, M. \& Hedges, L. V. (2006). Preschool children's mathematical knowledge: The effect of teacher "math talk“. Developmental Psychology, 42, 59-69. https://dx.doi. org/10.1037/0012-1649.42.1.59

KMK (2004). Standards für die Lehrerbildung: Bildungswissenschaften. Beschluss vom 16.12.2004. Zugriff am 23. 8. 2018 unter https://www.kmk.org/fileadmin/ veroeffentlichungen_beschluesse/2004/2004_12_16Standards-Lehrerbildung.pdf
KMK (2008). Ländergemeinsame inhaltliche Anforderungen für die Fachwissenschaften und Fachdidaktiken in der Lehrerbildung. Beschluss vom 16.10.2008. Zugriff am 23. 8. 2018 unter https://www.kmk.org/fileadmin/ Dateien/veroeffentlichungenbeschluesse/2008/2008_ 10_16-Fachprofile-Lehrerbildung.pdf

Kuhn, D., Iordanou, K., Pease, M. \& Wirkala, C. (2008). Beyond control of variables: What needs to develop to achieve skilled scientific thinking? Cognitive Development, 23, 435-451. https://dx.doi.org/10.1016/j.cog dev.2008.09.006

Leseman, P. P. M., Scheele, A. F., Mayo, A. Y. \& Messer M.H. (2007). Home literacy as a special language environment to prepare children for school. Zeitschrift für Erziehungswissenschaft, 10, 334-355. https://dx. doi.org/10.1007/s11618-007-0040-9

Leuchter, M. \& Saalbach, H. (2014). Verbale Unterstützungsmaßnahmen im Rahmen eines naturwissenschaftlichen Lernangebots in Kindergarten und Grundschule. Unterrichtswissenschaft, 42,117-131.

Lidstone, J., Meins, E. \& Fernyhough, C. (2010). The roles of private speech and inner speech in planning during middle childhood: Evidence from a dual task paradigm. Journal of Experimental Child Psychology, 107 438-451. https://dx.doi.org/10.1016/j.jecp.2010.06. 002

Lo, Y.Y. \& Lo, E.S.C. (2014). A meta-analysis of the effectiveness of English-medium education in Hong Kong. Review of Educational Research, 84, 47-73. https:// dx.doi.org/10.3102/0034654313499615

Loewenstein, J. \& Gentner, D. (1998). Relational language facilities analogy in children. In M.A. Gernsbacher \& S. H. Derry (Eds.), Proceedings of the twentieth annual conference of the cognitive science society (pp. 615-620). Mahwah, NJ: Erlbaum.

Lupyan, G. (2012). What do words do? Towards a theory of language-augmented thought. In B. H. Ross (Ed.), The psychology of learning and motivation (Vol. 57, pp. 255-297). Academic Press. https://dx.doi.org/10.10 16/B978-0-12-394293-7.00007-8

Lütke, B., Petersen, I. \& Tajmel, T. (Hrsg.). (2017). Fachintegrierte Sprachbildung: Forschung, Theoriebildung und Konzepte für die Unterrichtspraxis (DaZ-Forschung, Bd. 8). Berlin: De Gruyter. https://dx.doi.org/10.1515/ 9783110404166

Mannel, S., Hardy, I., Sauer, S. \& Saalbach, H. (2016) Sprachliches Scaffolding zur Unterstützung naturwissenschaftlichen Lernens im Kindergartenalter. In E. Tschirner, O. Bärenfänger \& J. Möhring (Hrsg.), Deutsch als fremde Bildungssprache: Das Spannungsfeld von Fachwissen, Sprachlicher Kompetenz, Diagnostik und Didaktik (S.97-114). Tübingen: Stauffenburg.

Marian, V. \& Fausey, C. M. (2006). Language-dependent memory in bilingual learning. Applied Cognitive Psychology, 20, 1025-1047. https://dx.doi.org/10.1002/ acp. 1242

Mascareño, M., Snow, C.E., Deunk, M. I. \& Bosker, R. J. (2016). Language complexity during read-alouds and kindergartners' vocabulary and symbolic understanding. Journal of Applied Developmental Psychology, 44, 39-51. https://dx.doi.org/10.1016/j.appdev.2016.02. 001

Medimorec, S., Pavlik, P. I., Olney, A., Graesser, A. C. \& Risko, E. F. (2015). The language of instruction: Compensating for challenge in lectures. Journal of Educational Psychology, 107, 971-990. https://dx.doi.org/ 10.1037/edu0000024 
Miller, K. F., Smith, C. M., Zhu, J. \& Zhang, H. (1995). Preschool origins of cross-national differences in mathematical competence: The role of number-naming systems. Psychological Science, 6, 56-60. https://dx. doi.org/10.1111/j.1467-9280.1995.tb00305.x

Miller, O.F. (1996). Linguistic structure and cognitive development: Chinese/English comparison. Studies in Linguistic Science, 26, 237-254.

Möller, J., Hohenstein, F., Fleckenstein, J. \& Baumert, J. (2017). Formen und Effekte des Fremdsprachener werbs und der bilingualen Beschulung. In J. Möller, F. Hohenstein, J. Fleckenstein, J. Baumert \& O. Köller (Hrsg.), Erfolgreich integrieren: Die staatliche EuropaSchule Berlin (S. 25-48). Münster: Waxmann.

Möller, J., Fleckenstein, J., Hohenstein, F., Preusler, S., Paulick, I. \& Baumert, J. (2018). Varianten und Effekte bilingualen Lernens in der Schule. Zeitschrift für Erziehungswissenschaft, 21, 4-28. https://dx.doi.org/10 $1007 /$ s11618-017-0791-x

Morek, M. \& Heller, V. (2012). Bildungssprache-Kommunikative, epistemische, soziale und interaktive Aspekte ihres Gebrauchs. Zeitschrift für angewandte Linguistik, 57, 67-101. https://dx.doi.org/10.1515/zfal-2012-00 11

Morris-Lange, S., Wagner, K. \& Altinay, L. (2016). Lehrerbildung in der Einwanderungsgesellschaft: Qualifizierung für den Normalfall Vielfalt. Zugriff am 23. 8.2018 unter https://www.stiftung-mercator.de/media/downloads/ 3_Publikationen/SVR_Mercator_Institut_Policy_ Brief_Lehrerbildung_September_2016.pdf

Paetsch, J. \& Felbrich, A. (2015). Longitudinale Zusammenhänge zwischen sprachlichen Kompetenzen und elementaren mathematischen Modellierungskompetenzen bei Kindern mit Deutsch als Zweitsprache. Psychologie in Erziehung und Unterricht, 63, 16-33. https://dx.doi.org/10.2378/peu2016.art03d

Paetsch, J., Felbrich, A. \& Stanat, P. (2015). Der Zusammenhang von sprachlichen und mathematischen Kom petenzen bei Kindern mit Deutsch als Zweitsprache. Zeitschrift für Pädagogische Psychologie, 29, 19-29. https://dx.doi.org/10.1024/1010-0652/a000142

Perrone-Bertolottia, M., Rapin, L., Lauchaux, J.-P., Baciu, M. \& Løevenbruck, H. (2014). What is that little voice inside my head? Inner speech phenomenology, its role in cognitive performance, and its relation to self-monitoring. Behavioural brain research, 261, 220-239. https://dx.doi.org/10.1016/j.bbr.2013.12.034

Pica, P., Lemer, C., Izard, V. \& Dehaene, S. (2004). Exact and approximate arithmetic in an amazonian indigene group. Science, 306, 499-503. https://dx.doi.org/10. $1126 /$ science. 1102085

Piesche, N., Jonkmann, K., Fiege, C. \& Keßler, J.-U. (2016). CLIL for all? A randomised controlled field experiment with sixth-grade students on the effects of content and language integrated science learning. Learning and Instruction, 44, 108-116. https://dx.doi.org/10.1016/ j.learninstruc.2016.04.001

Pimm, D. (1987). Speaking mathematically. London: Routledge.

Pöhlmann, C., Haag, N. \& Stanat, P. (2013). Zuwanderungsbezogene Disparitäten. In H.A. Pant, P. Stanat, U. Schroeders, A. Roppelt, T. Siegle \& C. Pöhlmann (Hrsg.), IQB Ländervergleich 2012: Mathematische und naturwissenschaftliche Kompetenzen am Ende der Sekundarstufe I (S. 279-329). Münster: Waxmann.
Prediger, S., Wilhelm, N., Büchter, A., Gürsoy, E. \& Benholz, C. (2015). Sprachkompetenz und Mathematikleistung - Empirische Untersuchung sprachlich bedingter Hürden in den Zentralen Prüfungen 10 Journal für Mathematik-Didaktik, 36, 77-104. https:// dx.doi.org/10.1007/s13138-015-0074-0

Renkl, A. (2009). Wissenserwerb. In E. Wild \& J. Möller (Hrsg.), Pädagogische Psychologie (S. 3-26). Berlin: Springer. https://dx.doi.org/10.1007/978-3-540-885 73-3_1

Reusser, K. (2001). Co-constructivism in educational theory and practice. In N.J. Smelser \& B. Baltes (Eds.), International Encyclopedia of the Social and Behavioral Sciences (20th ed., pp. 2058-2062). München: Elsevier. https://dx.doi.org/10.1016/B0-08-043076-7/02 408-6

Rincke, K. (2010). Alltagssprache, Fachsprache und ihre besonderen Bedeutungen für das Lernen. Zeitschrift für Didaktik der Naturwissenschaften, 16, 235-260.

Rincke, K. (2011). It's rather like learning a language: Development of talk and conceptual understanding in mechanics lessons. International Journal of Science Education, 33, 229-258. https://dx.doi.org/10.1080/ 09500691003615343

Roth, W.-M. \& Duit, R. (2003). Emergence, flexibility, and stabilization of language in a physics classroom. Journal of Research in Science Teaching, 40, 869-897. https://dx.doi.org/10.1002/tea.10114

Roussel, S., Joulia, D., Tricot, A. \& Sweller, J. (2017). Learning subject content through a foreign language should not ignore human cognitive architecture: A cognitive load theory approach. Learning and Instruction, 52, 69-79. https://dx.doi.org/10.1016/j.learninstruc. 20 17.04 .007

Rumlich, D. (2016). Evaluating bilingual education in Germany: CLIL students' general English proficiency, EFL self-concept and interest (Mehrsprachigkeit in Schule und Unterricht, Bd. 15). Frankfurt a. M.: Peter Lang https://dx.doi.org/10.3726/978-3-653-06460-5

Saalbach, H., Eckstein, D., Andri, N., Hobi, R. \& Grabner, R. H. (2013). When language of instruction and language of application differ: Cognitive costs of bilingual mathematics learning. Learning and Instruction, 26, 36-44. https://dx.doi.org/10.1016/j.learninstruc. 20 13.01 .002

Saalbach, H., Gunzenhauser, C., Kempert, S. \& Karbach, J. (2016). Der Einfluss von Mehrsprachigkeit auf mathematische Fähigkeiten bei Grundschulkindern mit niedrigem sozioökonomischen Status. Frühe Bildung, 5, 73-81. https://dx.doi.org/10.1026/2191-9186/a000 255

Saalbach, H., Imai, M. \& Schalk, L. (2012). Grammatical gender and inferences about biological properties in german-speaking children. Cognitive Science, 36, $1251-$ 1267. https://dx.doi.org/10.1111/j.1551-6709.2012. 01251.x

Saji, N., Imai, M., Saalbach, H., Zhang, Y., Shu, H. \& Okada, H. (2011). Word learning does not end at fast-mapping: Evolution of verb meanings through reorganization of an entire semantic domain. Cognition, 118, 45-61. https://dx.doi.org/10.1016/j.cognition.2010. 09.007

Schalk, L., Saalbach, H. \& Stern, E. (2016). Approaches to foster transfer of formal principles: Which route to take? PloS one, 11 (2), e0148787. https://dx.doi.org/ 10.1371 /journal.pone.0148787 
Schleppegrell, M. J. (2004). The language of schooling: $A$ functional linguistics perspective. New York, NY: Routledge.

Schleppegrell, M. J. (2007). The linguistic challenges of mathematics teaching and learning: A research review. Reading \& Writing Quarterly, 23, 139-159. https:// dx.doi.org/10.1080/10573560601158461

Schleppegrell, M.J. (2010). Language in mathematics teaching and learning. In J. N. Moschkovich (Ed.), Research in mathematics education. Language and mathematics education: Multiple perspectives and directions for research (pp. 73-112). Charlotte, NC: Information Age Publishing.

Service, F. \& Kohonen, V. (1995). Is the relation between phonological memory and foreign language learning accounted for by vocabulary acquisition? Applied Psycholinguistics, 16, 155-172. https://dx.doi.org/10.10 $17 /$ S0142716400007062

Snow, C.E. (2010). Academic language and the challenge of reading for learning about science. Science, 328 450-452. https://dx.doi.org/10.1126/science.11825 97

Snow, C. E. \& Uccelli, P. (2009). The challenge of academic language. In N. Torrance \& R. D. Olson (Eds.), The Cambridge handbook ofliteracy (pp. $112-133$ ). New York, NY: Cambridge University Press. https://dx.doi. org/10.1017/CBO9780511609664.008

Spelke, E. S. \& Tsivkin, S. (2001). Language and number: A bilingual training study. Cognition, 78, 4588. https://dx.doi.org/10.1016/S0010-0277(00)0010 8-6

Tarelli, I., Schwippert, K. \& Stubbe, T. C. (2012). Mathematische und naturwissenschaftliche Kompetenzen von Schülerinnen und Schülern mit Migrationshintergrund. In W. Bos, H. Wendt, O. Köller, C. Selter \& T.C. Stubbe (Hrsg.), TIMSS 2011: Mathematische und naturwissenschaftliche Kompetenzen von Grundschulkindern in Deutschland im internationalen Vergleich (S. 247-268). Münster: Waxmann.

Tomasello, M. (1999). The cultural origins of human cognition. Cambridge, MA: Harvard University Press.

Vallotton, C. \& Ayoub, C. (2011). Use your words: The role of language in the development of toddlers' selfregulation. Early Childhood Research Quarterly, 26, 169 - 181. https://dx.doi.org/10.1016/j.ecresq.2010. 09.002

van de Pol, J., Volman, M. \& Beishuizen, J. (2010). Scaffolding in teacher-student interaction: A decade of research. Educational Psychology Review, 22, 271-296. https://dx.doi.org/10.1007/s10648-010-9127-6

van Dijk, T.A. \& Kintsch, W. (1983). Strategies of discourse comprehension. New York, NY: Academic Press.

Volmer, E., Grabner, R. H. \& Saalbach, H. (2018). Language switching costs in bilingual mathematics learning. Transfer effects and individual differences. Zeitschrift für Erziehungswissenschaft, 21, 71-96. https://dx.doi.org/ 10.1007/s11618-017-0795-6

Vygotsky, L.S. (1987). Thinking and speech: The collected works of Lev Vygotsky. New York, NY: Plenum.

Vygotsky, L. S. (2012). Thought and language (revised and expanded edition). Cambridge, MA: MIT.
Waxman, S. R. \& Markow, D. B. (1995). Words as invitations to form categories: Evidence from 12- to 13-monthold infants. Cognitive Psychology, 29, 257-302. https:// dx.doi.org/10.1006/cogp.1995.1016

Weinert, S. (2010). Beziehungen zwischen Sprachentwicklung und Gedächtnisentwicklung. In H.P. Trolldenier, W. Lenhard \& P. Marx (Hrsg.), Brennpunkte der Gedächtnisforschung: Entwicklungs- und pädagogisch-psychologische Perspektiven (S. 147-170). Göttingen: Hogrefe.

Weinert, S. (2016). Natürliche Sprache(n) und Formelsprache(n) in der Bildung. In J. Kilian, B. Brouër \& D. Lüttenberg (Hrsg.), Handbuch Sprache in der Bildung (S. 3-24). Berlin: De Gruyter. https://dx.doi. org/10.1515/9783110296358-003

Winsler, A. (2009). Still talking to ourselves after all these years: A review of current research on private speech In A. Winsler, C. Fernyhough \& I. Montero (Eds.), Private speech, executive functioning, and the development of verbal self-regulation (pp. 3-41). Cambridge, UK: Cambridge University Press.

Winsler, A. \& Naglieri, J. A. (2003). Overt and covert verbal problem-solving strategies: Developmental trends in use, awareness, and relations with task performance in children ages 5 to 17. Child Development, 74, 659678. https://dx.doi.org/10.1111/1467-8624.00561

Zaunbauer, A. C. M., Gebauer, S. K. \& Möller, J. (2012) Englischleistungen immersiv unterrichteter Schülerinnen und Schüler. Unterrichtswissenschaft, 4, 315-332.

Zaunbauer, A. C. M. \& Möller, J. (2010). Schulleistungsentwicklung immersiv unterrichteter Grundschüler in den ersten zwei Schuljahren. Psychologie in Erziehung und Unterricht, 57, 30-45. https://dx.doi.org/10.23 78/peu2010.art03d

\section{Prof. Dr. Sebastian Kempert}

Universität Potsdam

Strukturbereich Bildungswissenschaften Juniorprofessur für Empirische Grundschulpädagogik und -didaktik

\section{Karl-Liebknecht-Str. 23-24}

14176 Potsdam/Golm

E-Mail: sebastian.kempert@uni-potsdam.de

\section{Dr. Lennart Schalk}

PH Schwyz

Forschungsprofessur für MINT-Fachdidaktik

Zaystr. 42

6410 Goldau

E-Mail: lennart.schalk@phsz.ch

\section{Prof. Dr. Henrik Saalbach}

Universität Leipzig

Erziehungswissenschaftliche Fakultät

Professur für Pädagogische Psychologie

Marschnerstr. 31

04109 Leipzig

E-Mail: henrik.saalbach@uni-leipzig.de 